\title{
How Can One Choose the Best Method for Measuring FRET in a Microscope with My Biological System?
}

\author{
R. M. Clegg*, S. Breusegam**, N. Barry***, O. Holub****, Govindjee*, G. Redford* \\ * Physics Dept., 1110 W. Green Street, University of Illinois, Urbana, IL 61801; ** \\ University of Colorado Health Sciences Center, Dept. of Medicine, 4200 East 9th \\ Avenue, C281, Denver, CO 80262; *** Division of Renal Diseases and Hypertension, \\ University of Colorado Health Sciences Center, Dept. of Medicine, 4200 East 9th \\ Avenue, C281, Denver, CO 80262; Laboratório de Biofísica Química de Proteínas, \\ Universidade Federal do Rio de Janeiro, Departamento de Bioquimica Medica/ ICB/ \\ CCS, Bloco H / 20. andar / sala 19, CEP: 21941/590 - Ilha do Fundão, Rio de Janeiro - \\ RJ - Brasil
}

Which method is the best for imaging FRET? This is a common question. It is simply answered if you have only one possibility for measuring FRET. However, even in this case it is best to be aware of the pitfalls, as well as the advantages, for the different methods. It can be difficult to quantify FRET in cells because of experimental artifacts and complexities in determining the necessary photophysical parameters. There are many reasons for these difficulties: photobleaching, distributions of distances, varying relative angles between the donor and acceptor, aggregation of the labeled macromolecules, lack of information about relative concentrations of the complementary FRET pairs in different locations, stability of the image during data acquisition, less that $100 \%$ labeling of the donor and acceptor, spatially varying index of refraction, $\mathrm{pH}$ and ionic strength, different extents of static or dynamic quenching. Every effect contributes uniquely and to a different extent to the various methods of FRET measurements. In this lecture we will give an overview of several methods commonly used for measuring FRET in a fluorescence microscope. They will be demonstrated, evaluated and compared on two systems: intermolecular FRET between CFP and YFP hybridized to integrins and integrin associated proteins in C. elegans, and measurements on photosynthetic systems in algae and intact plants under stress. These two examples are chosen because they demonstrate different and complementary experimental difficulties. Each method of measurement has advantages and disadvantages in the various measurement circumstances.

The efficiency of energy transfer is measured in either in steady state or time-resolved fluorescence measurements. Each mode of measurement can be carried out in several ways. Steady state fluorescence is most common because this corresponds to the equipment usually available in most laboratories. Time-resolved measurements are presently located in select laboratories, mostly where the investigators have experience with fluorescence lifetime measurements; however, the equipment is becoming available commercially, and it is expected that these methods of fluorescence lifetime imaging, FLI, will rapidly become more common.

We will present a very brief illustrative overview of the basic mechanism of FRET, emphasizing the different pathways of de-excitation from the excited state, which clearly demonstrate the coupling between the physical photophysical mechanisms.. Using this as 
a background as an introduction to the practical measurements, we will discuss a number of common effects that can interfere significantly with a straightforward analysis of a FRET measurement. The advantages and disadvantages of the methods as well as the experimental peculiarities of each method will be discussed and demonstrated. and out the necessity of measuring in different ways if this is possible.

1.R.M. Clegg, "Nuts and Bolts of Excitation Energy Migration and Energy Transfer" in Advances in Photosynthesis and Respiration, Chlorophyll a Fluorescence: A Signature of Photosynthesis, Springer, Series Editor: Govindjee (2004)

2.O. Holub et al, Fluorescence lifetime imaging (FLI) in real-time - a new technique in photosynthesis research, Photosynthetica 38(4) (2000) 581.

3.R.M. Clegg, et al, Fluorescence Lifetime-resolved Imaging. Measuring lifetimes in an image. Why do it? How to do it. How to interpret it, in Methods in Enzymology, Biophotonics; Part A (two-volume issue); Eds G. Marriot and I. Parker; (2003) Vol. 360 (361 A); Sect. C. Imaging Microscopy; Chap. 22; p. 509-542 (pp.680); ISBN 0-12182263-X; Academic Press, San Diego. 